TRANSACTIONS OF THE

AMERICAN MATHEMATICAL SOCIETY

Volume 185, November 1973

\title{
WEAKLY ALMOST PERIODIC FUNCTIONALS ON THE FOURIER ALGEBRA
}

\author{
BY \\ CHARLES F. DUNKL AND DONALD E. RAMIREZ
}

\begin{abstract}
The theory of weakly almost periodic functionals on the Fourier algebra is herein developed. It is the extension of the theory of weakly almost periodic functions on locally compact abelian groups to the duals of compact groups. The complete direct product of a countable collection of nontrivial compact groups furnishes an important example for some of the constructions.
\end{abstract}

\section{INTRODUCTION AND BACKGROUND}

Introduction. Eberlein used ergodic methods to create weakly almost periodic functions on groups [5]. In this paper we present a nonabelian extension of the theory. Our extension is not in the already explored direction of functions on groups, but rather we use the idea that the space of bounded measurable functions on a locally compact abelian (l.c.a.) group $\hat{G}$ is the dual of the Fourier algebra of $G$ (the dual (1.c.a.) group of $\hat{G}$ ), where the Fourier algebra of $G$ is the set of Fourier transforms of the integrable functions on $\hat{G}$. The Fourier algebra is defined on each locally compact group, and has been investigated by Eymard [6]. It is a commutative Banach algebra of continuous functions on $G$ vanishing at infinity. Its dual turns out to be the von Neumann algebra generated by the left translations on the Hilbert space of square-integrable (left Haar measure) functions on the group. This algebra of operators is a module over the Fourier algebra, and we use module-type definitions of weakly almost periodic (w.a.p.) elements of it.

For locally compact group $G$ with Fourier algebra $A(G)$, let $W(\hat{G})$ be the set of $\phi \in A(G)^{*}$ (the dual of $A(G)$ ) such that the map $f \mapsto f \cdot \phi$ of $A(G) \rightarrow A(G)^{*}$ is a weakly compact operator $(f \cdot \phi$ is the module action). Many of the classical theorems on w.a.p. functions also hold in this setting of w.a.p. functionals on the Fourier algebra. For example, $W(\hat{G})$ is a Banach space with a unique invariant (in a sense to be defined) mean, and there is a monomorphism (the FourierStieltjes transform 7 ) of $M(G)$, the measure algebra of $G$, into $W(\hat{G})$.

If $G$ is compact then the easily accessible structure of the dual of $G$ allows a more detailed study. The dual of $A(G)$ is an algebra of bounded matrix-valued functions on a discrete space, and we are able to use the idea of quasi-uniform

Received by the editors September 7, 1970.

AMS (MOS) subject classifications (1970). Primary 43A10, 43-00, 43A75; Secondary 22D40, $43 \mathrm{~A} 07$.

Key words and phrases. Weakly almost periodic, almost periodic, means, quasi-uniform convergence, Fourier algebra. 
convergence (invented for use on scalar valued functions) to characterize weak compactness. This technique allows us to show that if $G$ is the complete direct product of a countable collection of nontrivial compact groups, then $\Im M(G)$ is not dense in $W(\hat{G})$, and $W(\hat{G})$ is a proper closed subspace of $A(G)^{*}$.

Here are some details on the organization of this paper. It is split into sections and chapters.

\$I contains the present material and Chapter 1 dealing with the classical abelian theory from the $A(G)$-module point of view.

$\S I$ is the main part of the paper, and deals with compact groups. Chapter 2 gives the basic definitions and properties of the w.a.p. functionals and the mean, and uses the ergodic semigroup theory of Eberlein [5]. Chapter 3 deals with quasiuniform convergence, and heavily uses the theory of weak topologies on Banach spaces. In Chapter 4 , the $T$-sets are constructed. These are "thin" subsets of the dual of $G$, which have the property that any $\phi \in A(G)^{*}$ which is carried by one, is w.a.p. Chapters 5 and 6 show, for the complete direct product $G$ of a countable collection of nontrivial compact groups, that $\exists M(G)$ is not dense in $W(\hat{G})$ and that $W(\hat{G}) \neq A(G)^{*}$.

§III contains Chapter 7 dealing with almost periodic functionals, and Chapter 8, which is a swift extension of the material of Chapter 2 and 7 to all locaily compact groups.

Notation is cumulative throughout the paper. In $\S I I, G$ denotes a compact group, except when, for purposes of illustration, we consider 1.c.a. groups.

During the preparation of this paper the authors were partly supported by NSF grant GP-8981.

Chapter 1. Abelian background. In this section, we will let $G$ denote a locally compact abelian (l.c.a.) group with $\hat{G}$ its dual group. An essentially bounded Borel function $\phi$ on $G$ is said to be weakly almost periodic (w.a.p.) if and only if the set of translates of $\phi$, denoted by $O(\phi)$, is relatively weakly compact in $L^{\infty}(G)$, the space of all essentially bounded Borel functions on $\hat{G}$ (see [5]). We will first give an equivalent definition (for w.a.p. functions) which will allow us to develop the theory of w.a.p. functions on duals of nonabelian groups.

We let $L^{\prime}(G)$ be the Banach algebra of integrable functions on $G$ with convolution * as the multiplication. The Fourier transform $\exists$ takes $L^{\prime}(G)$ into $C^{B}(\hat{G})$, the space of continuous bounded functions on $\hat{G}$. The Fourier algebra of $G, A(G)$, consists of those continuous functions $f$ on $G$ for which $\exists f \in L^{1}(\hat{G})$. The space $A(G)$ is a Banach algebra under the pointwise operations and the norm $\|f\|_{A}=\|\Im f\|_{1}$. Now since $A(G) \cong L^{1}(\hat{G})$, the dual of $A(G)$ can be identified with $L^{\infty}(\hat{G})$, and the pairing is given by $\langle f, \phi\rangle=\int_{G}(\exists f)(y) \phi(y) d m_{G}(y), f \in A(G)$, $\phi \in L^{\infty}(\hat{G})$, and where $m_{\hat{G}}$ is the Haar measure on $\hat{G}$.

We will now need the fact that $L^{\infty}(\hat{G})$ is a Banach module (see [6], [7]) over $A(G)$ : given $f \in A(G), \phi \in L^{\infty}(\hat{G})$, define $f \cdot \phi \in L^{\infty}(\hat{G})$ by $\langle g, f \cdot \phi\rangle=\langle f g$, $\phi\rangle, g \in A(G)$. 
Fix $\phi \in L^{\infty}(\hat{G})$. Let $T$ be the bounded operator from $A(G)$ to $L^{\infty}(\hat{G})$ given by $f \mapsto f \cdot \phi, f \in A(G)$. Note $\|f \cdot \phi\|_{\infty} \leq\|f\|_{A}\|\phi\|_{\infty}$. Now for $g \in A(G)$,

$$
\langle g, f \cdot \phi\rangle=\langle f g, \phi\rangle=\int_{G} \mathcal{Y}(f g)(y) \phi(y) d m_{G}(y)=\left\langle g,(\exists f)^{\bullet} * \phi\right\rangle
$$

where $\check{h}(y)=h(-y), h \in L^{\infty}(\hat{G})$. Thus $T f=f \cdot \phi=(\exists f)^{\circ} * \phi$ which is in $C^{B}(\hat{G})$. We now use the theory of the representation of operators with values in a commutative $C^{*}$-algebra (see $[4$, p. 490]). The operator $T$ induces an adjoint map $\tau$ from $\beta \hat{G}$, the Stone-Čech compactification of $\hat{G}$, into $L^{\infty}(\hat{G})$ by the rule $\langle f, \tau(y)\rangle=T f(y), f \in A(G), y \in \beta \hat{G}$. Thus for $f \in A(G), y \in \hat{G} \subset \beta \hat{G}$, we have $\langle f, \tau(y)\rangle=(\check{f} * \phi)(y)=\langle f, R(y) \phi\rangle$ where $(R(y) \psi)(z)=\psi(y+z), \psi$ $\in L^{\infty}(\hat{G}), z \in \hat{G}$. Hence $\tau(y)=R(y) \phi$ for $y \in \hat{G}$.

Now $\tau: \beta \hat{G} \rightarrow L^{\infty}(\hat{G})$ is always continuous in the weak-* topology $\sigma\left(L^{\infty}(\hat{G}), L^{1}(\hat{G})\right)$. Further, $T$ is a weakly compact operator if and only if $\tau$ is continuous in the weak topology $\sigma\left(L^{\infty}(\hat{G}), L^{\infty}(\hat{G})^{*}\right)$ (for $X$ a normed space, $X^{*}$ denotes the dual of $X$ ). Now if $T$ is a weakly compact operator, then it follows that $O(\phi)$ is relatively weakly compact. Conversely, if $O(\phi)$ is relatively weakly compact, it follows by standard point-set topology methods that $\tau$ is continuous into $L^{\infty}(\hat{G})$ with the weak topology. Thus we have shown the following theorem.

Theorem 1.1. Let $G$ be a locally compact abelian group. For $\phi \in L^{\infty}(\hat{G})$ to be weakly almost periodic it is necessary and sufficient that the map $f \mapsto f \cdot \phi$ from $A(G)$ to $C^{B}(\hat{G})(f \cdot \phi=\breve{f} * \phi)$ is a weakly compact operator.

Remark 1.2. Choose an approximate identity $\left\{u_{\lambda}\right\}$ in $L^{1}(\hat{G})$ (G is an 1.c.a. group) such that $\check{u}_{1}=u_{\lambda}$ and $\left\|u_{\lambda}\right\|_{1}=1$ for each $\lambda$. Then for any $\phi \in L^{\infty}(\hat{G})$ we have that $u_{\lambda} \cdot \phi=\check{u}_{\lambda} * \phi$ converges weak-* to $\phi$. Each $u_{\lambda} \cdot \phi \in C_{u}^{B}(\hat{G})$, the space of uniformly continuous bounded functions on $G$. Now suppose further that $\phi$ is w.a.p.; then $O(\phi)$ is relatively weakly compact. Thus $\left\{u_{\lambda} \cdot \phi\right\}$ has a weak cluster point in $\overline{O(\phi)} \subset C_{u}{ }^{B}(\hat{G})$ (recall for convex sets, the norm closure is the weak closure), which must be $\phi$. This proves the following:

Theorem 1.3. If $\phi \in L^{\infty}(\hat{G})$ and is w.a.p., and $G$ is l.c.a., then $\phi$ is uniformly continuous.

\section{COMPACT GROUPS}

Chapter 2. Basic definitions and the mean. Let $G$ be a compact nonabelian group. Using our previous notation [2, Chapter 7], we let $G$ denote the set of equivalence classes of continuous unitary irreducible representations of $\hat{G}$. For $\alpha \in \hat{G}$, let $T_{\alpha}$ be an element of $\alpha$. Then $T_{\alpha}$ is a homomorphism of $G$ into $U\left(n_{\alpha}\right)$, the group of $n_{\alpha} \times n_{\alpha}$ unitary matrices where $n_{\alpha}$ is the dimension of $\alpha$. We use $T_{\alpha}(x)_{i j}$ to denote the matrix entries of $T_{\alpha}(x), 1 \leq i, j \leq n_{\alpha}$, and $T_{\alpha i j}$ to denote the function $x \mapsto T_{\alpha}(x)_{i j}$. Now $T_{\alpha}(x y)_{i j}=\sum_{k=1}^{n_{\alpha}} T_{\alpha}(x)_{i k} T_{\alpha}(y)_{k j}$ and $T_{\alpha}\left(y^{-1}\right)_{i j}=\overline{T_{\alpha}(y)_{j i}}$. Furthermore, $T_{a i j} \in C(G)$, the space of continuous functions on $G$. For $\alpha \in \hat{G}$, 
let $\chi_{\alpha}(x)=\operatorname{trace}\left(T_{\alpha}(x)\right)=\sum_{i=1}^{n_{a}} T_{\alpha}(x)_{i i}$. The function is called the character of $\alpha$ and it is independent of the choice of $T_{\alpha}$ in $\alpha$.

Let $X$ be an $n$-dimensional complex inner product space. Let $\mathcal{B}(X)$ be the space of linear maps from $X \rightarrow X$. We define the operator norm of $A \in \mathcal{B}(X)$ by $\|A\|_{\infty}=\sup \{|A \xi|: \xi \in X,|\xi| \leq 1\}$. The trace of $A, \operatorname{Tr} A$, is $\sum_{i=1}^{n}\left(A \xi_{i}, \xi_{i}\right)$ where $\left\{\xi_{i}\right\}_{i=1}^{n}$ is any orthonormal basis for $X$ and $(\cdot, \cdot)$ denotes the inner product in $X$. Let $|A|$ denote $\left(A^{*} A\right)^{1 / 2}$. The value $\|A\|_{\infty}$ is the spectral radius of $|A|$; i.e. $\max \left\{\lambda_{i}: 1 \leq i \leq n\right\}$ where $\lambda_{i}$ are the eigenvalues of $|A|$.

Let $\phi$ be a set $\left\{\phi_{\alpha}: \alpha \in \hat{G}\right.$ where $\left.\phi_{\alpha} \in \mathcal{B}\left(C^{n_{\alpha}}\right)\right\}$ such that $\sup \left\{\left\|\phi_{\alpha}\right\|_{\infty}: \alpha \in \hat{G}\right\}$ $<\infty$. The set of all such $\phi$ is denoted by $\mathcal{L}^{\infty}(\hat{G})$. It is a Banach algebra under the norm $\|\phi\|_{\infty}=\sup \left\{\left\|\phi_{\alpha}\right\|_{\infty}: \alpha \in \hat{G}\right\}$ and coordinatewise operations. Let $M(G)$ denote the measure algebra of $G$, that is, the space of finite regular Borel measures on $G$ with convolution as the multiplication. For $\mu \in M(G)$, the Fourier-Stieltjes transform of $\mu, \hat{\mu}$, is a matrix-valued function defined for $\alpha \in \hat{G}$ by $\alpha \mapsto \hat{\mu}_{\alpha}=\int_{G} T_{a}\left(x^{-1}\right) d \mu(x)$. Note that $\hat{\mu} \in \mathcal{L}^{\infty}(\hat{G})$. We sometimes write $\Im \mu$ for $\hat{\mu}$.

Let $A \in \mathcal{B}(X)$ where $X$ is a finite-dimensional complex inner product space. We define the dual norm to $\|\cdot\|_{\infty}$ by $\|A\|_{h}=\sup \left\{|\operatorname{Tr}(A B)|:\|B\|_{\infty} \leq 1\right\}$. This norm can be also characterized by $\|A\|_{1}=\operatorname{Tr}(|A|)$. For $\phi \in \mathcal{L}^{\infty}(\hat{G})$, we put $\|\phi\|_{h}=\sum_{\alpha \in G} n_{\alpha}\left\|\phi_{\alpha}\right\|_{h}$. Let $\mathcal{L}^{\prime}(\hat{G})=\left\{\phi \in \mathcal{L}^{\infty}(\hat{G}):\|\phi\|_{h}<\infty\right\}$. The space $\mathcal{L}^{\prime}(\hat{G})$ is a Banach space under the norm $\|\cdot\|_{\text {. For }} \phi \in \mathcal{L}^{\prime}(\hat{G})$, let $\operatorname{Tr}(\phi)$ $=\sum_{\alpha \in G} n_{\alpha} \operatorname{Tr}\left(\phi_{\alpha}\right)$.

We will now define $A(G)$, the Fourier algbra of $G$. Let $A(G)$ be the set of $f \in C(G)$ for which $\hat{f} \in \mathcal{L}^{\prime}(\hat{G})$. We norm $A(G)$ by $\|f\|_{A}=\|\Im f\|_{1}=\|\hat{f}\|_{\mathrm{h}}$ $=\sum_{\alpha \in G} n_{\alpha}\left\|\hat{f}_{\alpha}\right\|_{h}<\infty$. Note that $A(G)$ is isomorphic to $\mathcal{L}^{1}(\hat{G})$ by $f \mapsto \exists f$ because for any $\phi \in \mathcal{L}^{\prime}(\hat{G})$ the function $f(x)=\sum_{\alpha \in G} n_{\alpha} \operatorname{Tr}\left(\phi_{\alpha} T_{\alpha}(x)\right)$ is in $A(G)$; further,

$$
\|f\|_{\infty}=\sup \left\{\left|\sum_{\alpha \in G} n_{\alpha} \operatorname{Tr}\left(\phi_{\alpha} T_{\alpha}(x)\right)\right|: x \in G\right\} \leq \sum_{\alpha \in G} n_{\alpha}\left\|\phi_{\alpha}\right\|_{1}=\|\phi\|_{1} .
$$

We now recall the following facts (see [2, Chapter 8]): $A(G) \cong \mathcal{L}^{\prime}(\hat{G})$ by $f \mapsto \exists f, f \in A(G) ; A(G)$ is an algebra under pointwise multiplication; the dual of $\mathcal{L}^{\prime}(\hat{G})$ is $\mathcal{L}^{\infty}(\hat{G})$ and the correspondence is given by $\langle\psi, \phi\rangle=\operatorname{Tr}(\phi \psi)$, $\psi \in \mathcal{L}^{\prime}(\hat{G}), \phi \in \mathcal{L}^{\infty}(\hat{G})$. Thus the dual of $A(G)$ can be identified with $\mathcal{L}^{\infty}(\hat{G})$ and the pairing is given by $\langle f, \phi\rangle=\operatorname{Tr}(\phi \hat{f}), f \in A(G), \phi \in \mathcal{L}^{\infty}(\hat{G})$. The module action of $\mathcal{L}^{\infty}(\hat{G})$ over $A(G)$ is defined by the following: $f \in A(G)$, $\phi \in \mathcal{L}^{\infty}(\hat{G}), f \cdot \phi \in \mathcal{L}^{\infty}(\hat{G})$ by $\langle g, f \cdot \phi\rangle=\langle f g, \phi\rangle, g \in A(G)$. Note $\|f \cdot \phi\|_{\infty}$ $\leq\|f\|_{A}\|\phi\|_{b_{0}}$.

Definition 2.1. For $G$ a compact group and $\phi \in \mathcal{L}^{\infty}(\hat{G})$, we say that $\phi$ is weakly almost periodic (w.a.p.) if and only if the map $f \mapsto f \cdot \phi$ from $A(G)$ to $\mathcal{L}^{\infty}(\hat{G})$ is a weakly compact operator. The space of all such $\phi$ is denoted by $W(\hat{G})$. 
We denote the unit ball in $A(G)$ by $B$. We denote by $P$ the set of continuous positive definite functions on $G$ which at $e$, the identity of $G$, have the value 1 . Note that $P \subset A(G)$ and $f \in P$ if and only if $f \in B$ and $\hat{f}_{\alpha}$ is a positive matrix for each $\alpha \in \hat{G}$.

Proposition 2.2. For $\phi \in \mathcal{L}^{\infty}(\hat{G}), \phi \in W(\hat{G})$ if and only if $B \cdot \phi=\{f \cdot \phi: f$ $\in B\}$ is relatively weakly compact in $\mathcal{L}^{\infty}(\hat{G})$ if and only if $P \cdot \phi=\{f \cdot \phi: f \in P\}$ is relatively weakly compact in $\mathcal{L}^{\infty}(\hat{G})$.

Proof. We need only observe that any hermitian operator is a difference of two positive definite operators. Thus $B$ is contained in the balanced convex hull of $2 P$.

Definition 2.3. For $\phi \in \mathcal{L}^{\infty}(\hat{G})$, we define $O(\phi)$ to be the set $P \cdot \phi$. (Observe that $O(\phi)$ is convex.)

Remark 2.4. For $\phi \in \mathcal{L}^{\infty}(\hat{G})$, to show that $O(\phi)$ is relatively weakly compact it is necessary and sufficient to show that $O(\phi)$ is relatively weakly sequentially compact; equivalently, $O(\phi)$ is relatively weakly countably compact (see $[4, \mathrm{p}$. 430]).

Theorem 2.5. $W(\hat{G})$ is a closed *-subspace of $\mathcal{L}^{\infty}(\hat{G})$.

Proof. For $\phi \in \mathcal{L}^{\infty}(\hat{G})$, let $T: A(G) \rightarrow \mathcal{L}^{\infty}(\hat{G})$ be defined as before by $T f=f \cdot \phi, f \in A(G)$. Note that the operator norm of $T,\|T\|$, is the same as the sup norm of $\phi$ in $\mathcal{L}^{\infty}(\hat{G}),\|\phi\|_{\infty}$. Thus to show that $W(\hat{G})$ is a closed space we need only invoke [4, p. 438]: a uniform limit of weakly compact operators is weakly compact. The *-invariance follows since $O\left(\phi^{*}\right)=O(\phi)^{*}$ and $\phi \mapsto \phi^{*}$ is a weakly continuous map.

Theorem 2.6. $W(G)$ is a module over $A(G)$.

Proof. Let $\phi \in W(\hat{G})$ and $f \in A(G)$. We need to show that $f \cdot \phi \in W(\hat{G})$. We observe that $B \cdot(f \cdot \phi) \subset\left(\|f\|_{A} B\right) \cdot \phi$.

Definition 2.7. Let $M(\hat{G})$ be the closure of $\Im M(G)$ in $\mathcal{L}^{\infty}(\hat{G})$.

Theorem 2.8. The algebra $M(\hat{G})$ is contained in $W(\hat{G})$.

Proof. It will suffice to consider a positive measure $\mu \in M(G)$ with $\|\mu\|=1$. Let $\&$ be the Hilbert space $L^{2}(G, \grave{\mu})$. Consider the map $S$ : $\& \rightarrow \mathcal{L}^{\infty}(\hat{G})$ by $(g, S f)=\int(f g)^{\wedge} d \mu, g \in A(G), f \in$ \&. Now $|(g, S f)| \leq\|g\|_{\alpha}\|f\|_{\alpha} \leq\|g\|_{A}$ $\|f\|_{d,}$, and thus $S$ is strongly continuous and hence weakly continuous $[4$, p. 422]. Let $\left\{f_{n}\right\} \subset A(G),\left\|f_{n}\right\|_{A} \leq 1$. Then $\left\{f_{n}\right\}$ is contained in the unit ball of $\&$ which is weakly sequentially compact. Thus there is a subsequence $f_{n_{k}} \rightarrow^{k} h$ weakly in 4. Thus $S f_{n_{k}} \rightarrow^{k} S h$ weakly. It remains to observe that $S f=f \cdot \hat{\mu}, f \in A(G)$. This follows from:

$$
\begin{aligned}
(g, S f) & =\int(f g)^{-} d \mu=\operatorname{Tr}\left(\hat{\mu}(f g)^{\wedge}\right) \\
& =\langle f g, \hat{\mu}\rangle=\langle g, f \cdot \hat{\mu}\rangle, \quad f, g \in A(G) .
\end{aligned}
$$


Note that this implies that $C_{0}(\hat{G}) \subset W(\hat{G})$, where $C_{0}(\hat{G})=\{\phi$ $\in \mathcal{L}^{\infty}(\hat{G}):\left\|\phi_{a}\right\|_{\infty} \geq \varepsilon$ for only finitely many $\alpha \in \hat{G}$ for each $\varepsilon>0$ \}, since $C_{0}(\hat{G})$ is the closure of $\exists L^{\prime}(G)$ (see [2, Chapter 8]).

Recall that $P=\{f \in A(G): f$ is positive definite with $f(e)=1\}$. Observe that $P$ is a commutative semigroup of operators on $\mathcal{L}^{\infty}(\hat{G})$.

Definition 2.9. The semigroup $P$ is called ergodic (see $[5$, p. 220]) if it possesses at least one system of almost invariant integrals. By such a system one means a family of transformations $\left\{\rho_{\lambda}\right\}$ such that:

(1) $\rho_{\lambda}$ is a linear transformation of $\mathcal{L}^{\infty}(\hat{G})$ into itself,

(2) for each $\phi \in \mathcal{L}^{\infty}(\hat{G})$ and each $\lambda, \rho_{\lambda} \phi \in \overline{O(\phi)}$,

(3) $\sup _{\lambda}\left\|\rho_{\lambda}\right\|<\infty$,

(4) for each $f \in P, \lim _{\lambda}\left(f \rho_{\lambda}-\rho_{\lambda}\right) \phi=0$ and $\lim _{\lambda}\left(\rho_{\lambda} f-\rho_{\lambda}\right) \phi=0, \phi$ $\in \mathcal{L}^{\infty}(\hat{G})$.

Theorem 2.10. The semigroup $P$ is ergodic.

Proof. Let $\{\lambda\}$ be a neighborhood basis for $e \in G$. Let $u_{\lambda} \in P$ with support $u_{\lambda} \subset \lambda$. We order the net $\left\{u_{\lambda}\right\}$ by $u_{\lambda}>u_{k}$ if and only if $\lambda \subset \kappa$. Now since $W(\hat{G})$ is an $A(G)$-module (Theorem 2.5), we define the linear transformation $\rho_{\lambda}$ from $W(\hat{G})$ to $W(\hat{G})$ by $\rho_{\lambda} \phi=u_{\lambda} \cdot \phi, \phi \in W(\hat{G})$. Properties 2.9(1), 2.9(2), and 2.9(3) are immediate.

To show property 2.9(4) it will suffice to show for $f \in P$ that $\left\|f u_{\lambda}-u_{\lambda}\right\|_{A}$ $\rightarrow^{\lambda} 0$. It is therefore enough to show that $\left\|u_{\lambda} g\right\|_{A} \rightarrow^{\lambda} 0, g \in A(G)$ with $g(e)=0$. Now recall that each closed primary ideal in $A(G)$ is maximal (see [6, p. 229]). Thus given $g \in A(G)$ with $g(e)=0$ and $\varepsilon>0$, there is $h \in A(G)$ with $h=0$ on a neighborhood $V$ of $e$ such that $\|g-h\|_{A}<\varepsilon$. Now for $\lambda \subset V$, we have that $\left\|u_{\lambda} g\right\|_{A} \leq\left\|u_{\lambda}(g-h)\right\|_{A}+\left\|u_{\lambda} h\right\|_{A}<\varepsilon$.

Theorem 2.11 To every $\phi \in W(\hat{G})$ there corresponds a constant denoted by $\mathscr{I}(\phi)$ such that for $\phi, \psi \in W(\hat{G})$ one has

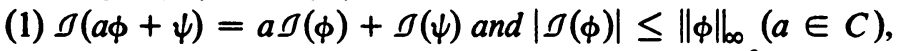

(2) $\mathscr{I}(I)=1$ (I denotes the identity operator in $\mathcal{L}^{\infty}(\hat{G})$ ),

(3) if $\phi_{\alpha} \geq 0$ for all $\alpha$, then $\supset(\phi) \geq 0$,

(4) $u_{\lambda} \cdot \phi \rightarrow^{\lambda} g(\phi) I$ in the norm of $\mathcal{L}^{\infty}(\hat{G}),\left\{u_{\lambda}\right\}$ as in 2.10,

(5) $\mathscr{\rho}\left(\phi^{*}\right)=\overline{I(\phi)}$

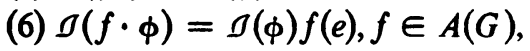

(7) properties (1), (2), and (6) uniquely determine $\subseteq$.

Proof. Since $P$ is an ergodic semigroup (Theorem 2.10), the ergodic theory (see [5, p. 220]) yields that each $\phi \in W(\hat{G})$ has a unique fixed point $F(\phi)$ in the weak closure of $O(\phi)$; i.e., $f \cdot F(\phi)=F(\phi)$, for each $f \in P$. We now argue that $F(\phi)$ is a constant multiple of the identity operator $I$ in $\mathcal{L}^{\infty}(\hat{G})$.

For $\psi \in \mathcal{L}^{\infty}(\hat{G})$ we define spt $\psi$ to be the intersection of all the compact sets $K$ in $G$ for which $f \cdot \psi=0, f \in A(G)$, whenever spt $f$ (the usual support) is 
contained in the complement of $K$. Further $\operatorname{spt}(f \cdot \phi) \subset \operatorname{spt} f \cap \operatorname{spt} \psi, f$ $\in A(G), \psi \in \mathcal{L}^{\infty}(\hat{G})$. So if $f \cdot \psi=\psi$, all $f \in P$, then spt $\psi \subset\{e\}$. Thus for $\phi \in W(\hat{G}), F(\phi)$ is a constant multiple of $I$ and we write $F(\phi)=g(\phi) I$ (see [6, Chapter 4]). Part (4) is from [5, p. 220]. The other claims follow from a standard argument.

Theorem 2.12. For $\mu \in M(G), \mathscr{D}(\hat{\mu})=\mu(\{e\})$.

Proof. Let $f \in P$, then $f \cdot \hat{\mu}=\mathcal{Y}(\check{f} d \mu)$. Thus as spt $f \rightarrow\{e\}, f \cdot \hat{\mu} \rightarrow \mu(\{e\}) I$.

Chapter 3. Quasi-uniform convergence. For $S$ a compact (Hausdorff) space, a sequence in $C(S)$ is weakly convergent if and only if it is bounded and quasiuniformly convergent on $S$ (see [4, p. 269]). Also for $S$ a locally compact space, a bounded sequence $\left\{f_{n}\right\}$ from $C^{B}(S)$ converges weakly to some $f \in C^{B}(S)$ if and only $f_{n} \rightarrow^{n} f$ pointwise on $S$ and every subsequence of $\left\{f_{n}\right\}$ converges quasiuniformly on $S$ to $f$ (see [4, p. 281]). That quasi-uniform convergence is useful in studying w.a.p. functions on 1.c.a. groups has been observed in [9]. We will develop similar results for $\mathcal{L}^{\infty}(\hat{G})$ (a compact group $G$ ). We will use the concept of quasi-uniform convergence which was introduced by Arzelà in 1884 for the unit interval.

In this chapter, $G$ denotes a compact group.

Definition 3.1. Let $\left\{\phi_{\lambda}\right\}$ be a net in $\mathcal{L}^{\infty}(\hat{G})$. One says that $\phi_{\lambda} \rightarrow^{\lambda} \phi, \phi$ $\in \mathcal{L}^{\infty}(\hat{G})$, quasi-uniformly on $\hat{G}$ if $\left(\phi_{\lambda}\right)_{\alpha} \rightarrow^{\lambda} \phi_{\alpha}$ for each $\alpha \in \hat{G}$ and for $\varepsilon>0$ and $\lambda_{0}$ there exists a finite number of indices $\lambda_{1}, \ldots, \lambda_{n} \geq \lambda_{0}$ such that, for each $\alpha \in \hat{G}, \min \left\{\left\|\left(\phi_{\lambda_{i}}\right)_{\alpha}-\phi_{\alpha}\right\|_{\infty}: 1 \leq i \leq n\right\}<\varepsilon$.

Definition 3.2. The unit ball in $A(G)$ is $B$. For $\alpha \in \hat{G}$, let $V_{\alpha}$ denote the (closed) span in $A(G)$ of $T_{\alpha i j}, 1 \leq i, j \leq n_{\alpha}$. We let $B_{\alpha}=B \cap V_{\alpha}$ and $E=\cup\left\{B_{\alpha}: \alpha\right.$ $\in \hat{G}\}$. The $A(G)$-closed convex hull of $E$ is denoted by $\overline{c o}(E)$.

Proposition 3.3. $B=\overline{c o}(E)$.

Proof. Let $f \in A(G)$ with $\|f\|_{A} \leq 1$. Thus $f$ has the Fourier series $\sum_{\alpha \in G} n_{\alpha} \tilde{f}_{\alpha}$ where $\tilde{f}_{\alpha}=f * \chi_{\alpha} \in V_{\alpha}$ and $\sum_{\alpha \in G} n_{\alpha}\left\|\tilde{f}_{\alpha}\right\|_{A}=\|f\|_{A}$ [2, Chapter 7].

Notation 3.4. Let $j$ denote the canonical map of $A(G)$ into $\mathcal{L}^{\infty}(\hat{G})^{*} \cong A(G)^{* *}$. The unit ball in $\mathcal{L}^{\infty}(\hat{G})^{*}$ will be denoted by $B^{* *}$. Let $\tau$ denote the weak-* topology on $\mathcal{L}^{\infty}(\hat{G})^{*}$. Now $j B$ is contained in and $\tau$-dense in $B^{* *}$ (see $[4$, p. 424]).

Proposition 3.5. $\overline{\operatorname{co}}^{\top}\left(\overline{j E}^{\tau}\right)=B^{* *}$.

Proof. It is immediate that $\overline{\operatorname{co}}^{\tau}\left(\overline{j E}^{\tau}\right)$ contains $j B$.

Theorem 3.6. Let $\left\{\phi_{n}\right\}$ be a sequence in $\mathcal{L}^{\infty}(\hat{G})$. If sup $\left\|\phi_{n}\right\|_{b_{\infty}}<\infty$ and $\left(\phi_{n}, f\right) \rightarrow^{n} 0, f \in \overline{j E}^{r}$ where $(\cdot, \cdot)$ denotes the natural pairing of $\mathcal{L}^{\infty}(\hat{G})$ and $\mathcal{L}^{\infty}(\hat{G})^{*}$, then $\phi_{n} \rightarrow^{n} 0$ weakly in $\mathcal{L}^{\infty}(\hat{G})$. 
Proof. Let $\left\{\phi_{n}\right\}$ be norm bounded and $\left(\phi_{n}, f\right) \rightarrow^{n} 0$ for $f \in \overline{j E}^{\tau}$. Now $\overline{j E}$ is a $\tau$-compact subset of $B^{* *}$. It will suffice to show that $\left(\phi_{n}, f\right) \rightarrow^{n} 0$ for $f \in B^{* *}$.

Now let $f \in B^{* *}$. Since $f$ is in the $\tau$-closed convex hull of $\overline{j E} \bar{E}^{\tau}$ (see Proposition 3.5), there exists a probability measure $\mu$ on $\overline{j E}^{\tau}$ which represents $f($ see $[8$, p. 5]). The result now follows from the Lebesgue dominated convergence theorem.

Theroem 3.7. Let $\left\{\phi_{n}\right\} \subset \mathcal{L}^{\infty}(\hat{G})$. If sup $\left\|_{\phi_{n}}\right\|_{\infty}<\infty$ and every subsequence $\left\{\phi_{n}\right\}$ converges quasi-uniformly on $\hat{G}$ to 0 , then $\phi_{n} \rightarrow^{n} 0$ weakly in $\mathcal{L}^{\infty}(\hat{G})$.

Proof. We may assume that $\left\|\phi_{n}\right\|_{\infty} \leq 1$ and suppose $\phi_{n} \nrightarrow^{n} 0$ weakly in

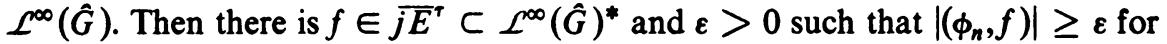
infinitely many $n$. By reindexing, we may assume that $\left|\left(\phi_{n}, f\right)\right| \geq \varepsilon$ for all $n$ (by passing to a subsequence).

Let $n_{1}, \ldots, n_{k}$ be such that $\min \left\{\left\|\left(\phi_{n_{i}}\right)_{\alpha}\right\|_{b_{\infty}}: 1 \leq i \leq k\right\}<\varepsilon$. Now letting $U_{i}=\left\{g \in \mathcal{L}^{\infty}(\hat{G})^{*}:\left|\left(\phi_{n_{i}}, g\right)\right|>\varepsilon\right\}, 1 \leq i \leq k$, we have that $f \in U=\bigcap\left\{U_{i}: 1\right.$ $\leq i \leq k\}$. Thus $U$ is a (nonempty) $\tau$-open neighborhood of $f$, and there is a $g \in A(G)$ such that $g \in E$ and $j g \in U$. But $E=\cup\left\{B_{\alpha}: \alpha \in \hat{G}\right\}$ and so $g$ is in some $B_{\alpha_{0}}$. Now $\left|\left(\phi_{n_{i}}, j g\right)\right|>\varepsilon, 1 \leq i \leq k$, contrary to the choice of $\left\{\phi_{n_{i}}: 1 \leq i\right.$ $\leq k\}$.

We now prove the converse.

Theorem 3.8. Let $\left\{\phi_{n}\right\} \subset \mathcal{L}^{\infty}(\hat{G})$. If $\left\{\phi_{n}\right\} \rightarrow^{n} 0$ weakly in $\mathcal{L}^{\infty}(\hat{G})$, then $\sup \left\{\left\|\phi_{n}\right\|_{\infty}\right\}<\infty$ and every subsequence of $\left\{\phi_{n}\right\}$ converges quasi-uniformly on $\hat{G}$ to 0.

Proof. Let $\varepsilon>0$ and $N \in Z^{+}$(positive integers) be given. For $f \in B^{* *}$, there is $n(f) \geq N$ such that $\left|\left(\phi_{n(f)}, f\right)\right|<\varepsilon$. Let $U(f)=\left\{g \in B^{* *}:\left|\left(\phi_{n(f)}, g\right)\right|<\varepsilon\right\}$, a $\tau$-open set. Now the collection of all $U(f), f \in B^{* *}$, covers $B^{* *}$; hence there is a finite subcover, $U\left(f_{1}\right), \ldots, U\left(f_{k}\right)$. Thus for $g \in B^{* *}, \min \left\{\left|\left(\phi_{n\left(f_{i}\right)}, g\right)\right|: 1 \leq i\right.$ $\leq k\}<\varepsilon$; and a fortiori, for $\alpha \in \hat{G}, \min \left\{\left\|\left(\phi_{m\left(f_{i}\right)}\right)_{\alpha}\right\|_{b_{\infty}}: 1 \leq i \leq k\right\}<\varepsilon$ (pairing against $B_{\alpha}$ ).

Remark 3.9. The above theorem is also valid for nets.

Chapter 4. $T$-sets. The concept of a $T$-set for an l.c.a. group has been useful in studying w.a.p. function on l.c.a. groups (for example see [9]). We will make the analogous definition of a $T$-set for the dual of a compact group.

Definition 4.1. For $\alpha, \beta \in \hat{G}$, one can consider the tensor product $T_{\alpha} \otimes T_{\beta}$ of the two representations. This tensor product decomposes into irreducible components: $T_{\alpha} \otimes T_{\beta} \cong \sum \bigoplus_{\gamma} m_{\alpha \beta}(\gamma) T_{\gamma}$, where $m_{\alpha \beta}(\gamma)=\int_{G} \chi_{\alpha} \chi_{\beta} \bar{\chi}_{\gamma} d m_{G}$, a nonnegative integer. For $E, F \subset \hat{G}$, we define $E \otimes F=\left\{\gamma \in \hat{G}: m_{\alpha \beta}(\gamma) \neq 0, \alpha \in E, \beta\right.$ $\in F\}$. This operation makes $\hat{G}$ into a hypergroup. If $E \otimes E \subset E$, then $E$ is called a subhypergroup of $\hat{G}$. For $\alpha \in \hat{G}$, there is a conjugate $\bar{\alpha} \in \hat{G}$ such that $\chi_{\bar{\alpha}}(x)=\overline{\chi_{\alpha}(x)}$, for each $x \in G$. If $E \subset \hat{G}$, then $\bar{E}=\{\bar{\alpha}: \alpha \in E\}$. We use $\{1\}$ to denote the trivial representation $G \rightarrow\{1\}$. 
Proposition 4.2. For $\alpha, \beta, \gamma \in \hat{G}, \quad m_{\alpha \beta}(\gamma)=m_{\beta \alpha}(\gamma)=m_{\alpha \bar{\gamma}}(\bar{\beta})=m_{\bar{\gamma} \beta}(\bar{\alpha})$ $=m_{\bar{\beta} \gamma}(\alpha)$. Also $\chi_{\alpha} \chi_{\beta}=\sum_{\gamma \in G} m_{\alpha \beta}(\gamma) \chi_{\gamma}$, thus $\sum_{\gamma \in G} m_{\alpha \beta}(\gamma) n_{\gamma}=n_{\alpha} n_{\beta}$.

Definition 4.3. Let $F \subset \hat{G}$. The set $F$ is said to be a $T$-set if and only if $(F \otimes \alpha) \cap(F \otimes \beta)$ is finite for all $\alpha, \beta \in \hat{G}$, with $\alpha \neq \beta$.

Theorem 4.4. Let $G=\Pi_{n} G_{n}$, the complete direct product of a countable collection of nontrivial compact groups $\left\{G_{n}\right\}$. Let $F_{n}$ be a finite subset of $\hat{G}_{n}$ such that $F_{n} \backslash\{1\} \neq \varnothing$. Under the natural identification of $\hat{G}_{n}$ with a subset of $\hat{G}$, the set $F=\cup_{n} F_{n}$ is an infinite $T$-set.

Proof. Now $\hat{G}$ is the hypergroup generated by $\cup_{n} \hat{G}_{n}$; that is, if $\alpha \in \hat{G}$, there exist $\alpha_{j} \in \hat{G}_{j}, \quad 1 \leq j \leq m<\infty$ ( $m$ depending on $\left.\alpha\right)$, such that $\chi_{\alpha}(x)$ $=\chi_{\alpha_{1}}\left(x_{1}\right) \cdots \chi_{\alpha_{m}}\left(x_{m}\right)$, where $x=\left(x_{1}, x_{2}, \ldots\right) \in G$ (equivalently, $\alpha=\alpha_{1} \otimes \cdots$ $\left.\otimes \alpha_{m}\right)$.

Let $\alpha, \beta \in \hat{G}, \alpha \neq \beta$. We write $\alpha=\alpha_{1} \otimes \cdots \otimes \alpha_{m}$ and $\beta=\beta_{1} \otimes \cdots \otimes \beta_{m}$, some $m \in Z_{+}$. We may assume that $\{1\} \notin F$. Let $F^{\prime}=\bigcup_{n=1}^{m} F_{n}$ and $F^{\prime \prime}$ $=\left(\cup_{n=m+1}^{\infty} F_{n}\right)$. If $\gamma, \gamma^{\prime} \in\left(\prod_{n=m+1}^{\infty} G_{n}\right)^{\wedge} \backslash\{1\}$, then $\alpha \otimes \gamma, \beta \otimes \gamma^{\prime}$ are irreducible representations of $G$ not in $\left(\prod_{n=1}^{m} G_{n}\right)^{\prime}$, and $\alpha \otimes \gamma \neq \beta \otimes \gamma^{\prime}$. Thus $F^{\prime \prime} \otimes \alpha$ and $F^{\prime \prime} \otimes \beta$ are disjoint sets and do not meet $\left(\prod_{n=1}^{m} G_{n}\right)^{\wedge}$. Further $F^{\prime} \otimes \alpha, F^{\prime} \otimes \beta$ are finite subsets of $\left(\prod_{n=1}^{m} G_{n}\right)^{\wedge}$. Thus $(F \otimes \alpha) \cap(F \otimes \beta)=\left(F^{\prime} \otimes \alpha\right) \cap\left(F^{\prime} \otimes \beta\right)$, a finite set.

Remark 4.5. The above results holds for the complete direct product of any infinite collection of compact groups. The proof is the same.

Definition 4.6. Let $\phi \in \mathcal{L}^{\infty}(\hat{G})$, define the carrier of $\phi$, cr $\phi$, to be the set $\left\{\alpha \in \hat{G}: \phi_{\alpha} \neq 0\right\}$. For $f \in A(G)$, we write $\operatorname{cr} f$ for $\operatorname{cr} \hat{f}$. (Observe for $\phi \in \mathcal{L}^{\infty}(\hat{G})$ that $\operatorname{cr} \phi \subset \hat{G}$ whereas spt $\phi \subset G$.) Note that $\operatorname{cr}(f g) \subset \operatorname{cr} f \otimes \operatorname{cr} g$, for $f, g$ $\in A(G)$.

Proposition 4.7. Let $f \in A(G)$ and $\phi \in \mathcal{L}^{\infty}(\hat{G})$. Then $\operatorname{cr}(f \cdot \phi) \subset(\overline{\operatorname{cr} f})$ $\otimes(\operatorname{cr} \phi)$.

Proof. Let $\alpha \in \hat{G}$. Then $\alpha \notin \operatorname{cr}(f \cdot \phi)$ if and only if $\langle g, f \cdot \phi\rangle=0$ for all $g \in V_{\alpha}$ if and only if $\langle f g, \phi\rangle=0$ for all $g \in V_{\alpha}$ if $\operatorname{cr}(f g) \cap \operatorname{cr}(\phi)=\varnothing$ if $(\operatorname{cr}(f) \otimes \alpha) \cap \operatorname{cr}(\phi)=\varnothing$ if and only if $m_{\alpha \beta}(\gamma)=0$ for all $\gamma \in \operatorname{cr}(\phi), \beta$ $\in \operatorname{cr}(f)$ if and only if $m_{\bar{\beta} \gamma}(\alpha)=0$ for all $\gamma \in \operatorname{cr}(\phi), \beta \in \operatorname{cr}(f)$, if and only if $\alpha \notin(\overline{\operatorname{cr} f}) \otimes(\operatorname{cr} \phi)$.

Theorem 4.8. Let $\phi \in \mathcal{L}^{\infty}(\hat{G})$ be such that $\mathrm{cr} \phi$ is a T-set in $\hat{G}$. Then $\phi \in W(\hat{G})$.

Proof. We first claim that it suffices to show that $U\left\{B_{\alpha} \cdot \phi: \alpha \in \hat{G}\right\}$ is relatively weakly (sequentially) compact; for then its norm closed convex hull, which is $\overline{B \cdot \phi} \supset \overline{O(\phi)}$ (Proposition 3.3) is also weakly compact by the KreinSmulian theorem (see [4, p. 434]). 
Choose a sequence $\left\{f_{n}\right\} \subset \cup B_{\alpha}$. Now if there is some $\alpha_{0} \in \hat{G}$ for which $f_{n} \in B_{\alpha_{0}}$ infinitely often, then by the compactness of $B_{\alpha_{0}}, f_{n} \cdot \phi$ has a weak cluster point. Thus we may suppose that no $B_{\alpha}$ contains more than one $f_{n}$. We will write $\psi_{n}=f_{n} \cdot \phi$ where $f_{n} \in B_{\alpha_{n}}$.

Suppose $\beta_{1}, \beta_{2} \in \hat{G}$ such that $\left(\psi_{n}\right)_{\beta_{1}} \neq 0$ and $\left(\psi_{n}\right)_{\beta_{2}} \neq 0$ for infinitely many $n$. Now $\beta \in \operatorname{cr} \psi_{n} \subset \bar{\alpha}_{n} \otimes \operatorname{cr} \phi$ only if $\alpha_{n} \in \bar{\beta} \otimes \operatorname{cr} \phi$. Thus $\left(\bar{\beta}_{1} \otimes \operatorname{cr} \phi\right) \cap$ $\left(\bar{\beta}_{2} \otimes \operatorname{cr} \phi\right)$ is an infinite set and so $\beta_{1}=\beta_{2}$.

Now let $\varepsilon>0$ and $N \in Z_{+}$. By the above remark $\lim _{n}\left(\psi_{n}\right)_{\alpha}=0$ for all $\alpha \in \hat{G}$ except at most one which we denote by $\delta$. By extracting a subsequence, we assume that $\lim _{n}\left(\psi_{n}\right)_{\alpha}=0$ for $\alpha \neq \delta$ and $\lim _{n}\left(\psi_{n}\right)_{\delta}=a \in \mathcal{B}\left(C^{n_{8}}\right)$. Let $\operatorname{cr} \psi_{N}$ $\cap \operatorname{cr} \psi_{N+1}=\left\{\beta_{3}, \ldots, \beta_{k}\right\} \subset \hat{G}$. Now choose $n_{3}, \ldots, n_{k} \geq N$ such that if $\beta_{i}$ $\neq \delta,\left\|\left(\psi_{n_{i}}\right)_{\beta_{i}}\right\|_{\infty}<\varepsilon, 3 \leq i \leq k$, and choose $n_{0} \geq N$ such that $\left\|\left(\psi_{n_{0}}\right)_{\delta}-a\right\|_{\infty}<\varepsilon$. Let $n_{1}=N$ and $n_{2}=N+1$. Letting $\Upsilon$ denote the operator in $\mathcal{L}^{\infty}(\hat{G})$ defined by $T_{\alpha}=0, \alpha \neq \delta, T_{\delta}=a$, we have that for any $\alpha \in \hat{G}, \min \left\{\left\|\left(\psi_{n_{i}}-\Upsilon\right)_{\alpha}\right\|_{\infty}: 0 \leq i\right.$ $\leq k\}<\varepsilon$. Thus $\left\{\psi_{n}\right\}$ converges quasi-uniformly on $\hat{G}$ to $\Upsilon$. Clearly any subsequence of $\left\{\psi_{n}\right\}$ does the same and so by Theorem 3.7, $\psi_{n} \rightarrow^{n} \Upsilon$ weakly. Consequently, we have that $O(\phi)$ is relatively weakly compact.

Chapter 5. A proper containment of $M(\hat{G})$ in $W(\hat{G})$. Recall from Definition 2.7 that $M(G)$ is the closure of $\exists M(G)$ in $\mathcal{L}^{\infty}(\hat{G})$. In Theorem 2.8 we showed that $\mathcal{O}(\hat{G})$ is contained in $W(\hat{G})$. We will, in this chapter, give examples of compact groups for which the containment is proper.

Now for $G$ 1.c.a., $M(G)^{\wedge-}$ has the following characterization [2]; for $\phi$ $\in C^{B}(\hat{G}), \phi \in M(G)^{--}$if and only if $\left\{f_{n}\right\} \subset A(G),\left\|f_{n}\right\|_{A} \leq 1, f_{n} \rightarrow^{n} 0$ pointwise on $G$ implies $\int_{G} \hat{f}_{n} \phi d m_{G} \rightarrow^{n} 0$. The nonabelian analogue also holds and has an identical proof. We state the result below.

Theorem 5.1. Let $G$ be a compact group and $\phi \in \mathcal{L}^{\infty}(\hat{G})$. The following are equivalent:

(A) $\phi \in \mathcal{M}(\hat{G})$,

(B) If a sequence $\left\{f_{n}\right\} \subset A(G),\left\|f_{n}\right\|_{A} \leq 1$, and $f_{n} \rightarrow^{n} 0$ pointwise on $G$, then $\left\langle f_{n}, \phi\right\rangle=\operatorname{Tr}\left(\hat{f}_{n} \phi\right) \rightarrow^{n} 0$.

Definition 5.2. For $G$ a compact group and $F \subset \hat{G}$, one says that $F$ is a central Sidon set if and only if for any $\phi \in \mathcal{L} \mathcal{L}(\hat{F})$ (the center of $\mathcal{L}^{\infty}(\hat{G}) \mid F$ ) there is a $\mu \in \mathcal{Z} M(G)$ (the center of $M(G)$ ) such that $\hat{\mu} \mid F=\phi$.

In [3], we studied central Sidon sets and showed for $G$ an infinite compact group that $\hat{G}$ is not a central Sidon set. It follows that $2 A(G)$ (the center of $A(G)$ relative to convolution) is proper in $\mathcal{Z C}(G)$ (the center of $C(G)$ relative to convolution). Thus for $\varepsilon>0$, there is $f \in \mathcal{Z} A(G)$ such that $\|f\|_{A}=1$ and $\|f\|_{\infty}<\varepsilon$. In fact, one may take $f$ to be a central trig series; i.e. $f(x)$ $=\sum_{\alpha \in F} c_{\alpha} \chi_{\alpha}(x)$, where $F$ is a finite subset of $\hat{G}$ and $c_{\alpha} \in C$.

Theorem 5.3. Let $G=\prod_{n} G_{n}$ be the complete direct product of a countable collection of nontrivial compact groups $\left\{G_{n}\right\}$. Then $\mathcal{M}(\hat{G})$ is a proper closed subspace of $W(\hat{G})$. 
Proof. Since $G$ is an infinite group, $\hat{G}$ is not a central Sidon set. Thus there is a central trig polynomial $f_{1}$ such that $\left\|f_{1}\right\|_{A}=1$ and $\left\|f_{1}\right\|_{\infty}<1$. Let $F_{1}=\operatorname{cr} f_{1}$.

Since cr $f_{1}$ is finite there is an $m_{1} \in Z_{+}$such that cr $f_{1} \subset H_{1}$ where $H_{1}$ $=\prod_{n=1}^{m_{1}} G_{n}$.

Now suppose that we have constructed central trig polynomials $f_{1}, \ldots, f_{k}$ and integers $1 \leq m_{1}<m_{2}<\cdots<m_{k}$ such that $\left\|f_{j}\right\|_{\infty}<1 / j$, $\left\|f_{j}\right\|_{A}=1$, and cr $f_{j}$ $\subset \hat{H}_{j}$, where $H_{j}=\prod_{n=m j+1}^{m_{j+1}} G_{n}$, for $j=1, \ldots, k$. Then there is a central trig polynomial $f_{k+1}$ on $K=\prod_{n=m_{k}+1}^{\infty} G_{n}$ such that $\left\|f_{k+1}\right\|_{A}=1$ and $\left\|f_{k+1}\right\|_{\infty}<$ $1 /(k+1)$ (observe that $f_{k+1}$ is also a trig polynomial on $G$ with the same sup and $A$ norms). Pick $m_{k+1}>m_{k}$ such that cr $f_{k+1} \subset\left(\prod_{n=m_{k}+1}^{m_{k+1}} G_{n}\right)^{\wedge}$. Thus by induction there exists a sequence of central trig polynomials $\left\{f_{k}\right\}$ on $G$ and a sequence of finite subgroups $\left\{H_{k}\right\}$ such that $G=\prod_{k=1}^{\infty} H_{k}$, and $\operatorname{cr} f_{k} \subset H_{k},\left\|f_{k}\right\|_{A}=1$, $\left\|f_{k}\right\|_{\infty}<1 / k$ for each $k \in Z_{+}$.

Now let $F=\bigcup_{k=1}^{\infty} \mathrm{cr} f_{k}$; then by Theorem $4.8, F$ is a $T$-set and so any $\phi \in \mathcal{L}^{\infty}(\hat{G})$ with $\operatorname{cr} \phi \subset F$ is in $W(\hat{G})$. Since each $f_{k}$ has $\left\|f_{k}\right\|_{A}=1$, there is $\phi_{k} \in \mathcal{Z} \mathcal{L}^{\infty}(\hat{G})$ such that $\operatorname{cr} \phi_{k}=\operatorname{cr} f_{k},\left\|\phi_{k}\right\|_{\infty}=1$ and $\left\langle f_{k}, \phi_{k}\right\rangle=1$. Let $\psi$ $\in \mathcal{Z} \mathcal{L}^{\infty}(\hat{G})$ be defined by $\psi_{\alpha}=\left(\phi_{k}\right)_{\alpha}$ if $\alpha \in \operatorname{cr} f_{k}$ for some $k$, and $\psi_{\alpha}=0$ otherwise. Thus $\psi \in W(\hat{G})$. Furthermore, since $\left\langle f_{k}, \psi\right\rangle=\left\langle f_{k}, \phi_{k}\right\rangle \nrightarrow^{k} 0$, we have $\psi \notin \mathcal{O}(\hat{G})$ (by Theorem 5.1).

Remark 5.4. Observe that the above result also holds for the complete direct product of an infinite collection of nontrivial compact groups. The proof is identical with the one above. Also our proof shows that $\mathcal{Z} M(\hat{G})$ is proper in $W(\hat{G}) \cap \mathcal{L} \mathcal{L}^{\infty}(\hat{G})$.

Remark 5.5. If $G$ is a nondiscrete l.c.a. group then the space of uniform limits on $\hat{G}$ of Fourier-Stieltjes transforms, $M(G)^{-}$, is a proper subspace of the continuous weakly almost periodic functions on $G$. The general result is due to Ramirez [9].

Remark 5.6. Let $M_{d}(G)$ denote the space of discrete measures on $G$ and $M_{d}(\hat{G})$ its closure in $\mathcal{L}^{\infty}(\hat{G})$. Just as $\mathcal{M}(\hat{G})$ has a characterization as a class of linear functionals on $A(G)$ (Theorem 5.1), $\circlearrowleft M_{d}(\hat{G})$ may be characterized similarly. The proof is similar to the abelian case (see [2, Chapter 3]). We present the following as a contrast to Theorem 5.1.

Theorem 5.7. Let $G$ be a compact group and $\phi \in \mathcal{L}^{\infty}(\hat{G})$. The following are equivalent:

(A) $\phi \in M_{d}(\hat{G})$.

(B) If a net $\left\{f_{\lambda}\right\} \subset A(G),\left\|f_{\lambda}\right\|_{A} \leq 1$, and $f_{\lambda} \rightarrow^{\lambda} 0$ pointwise on $G$, then $\left\langle f_{\lambda}, \phi\right\rangle=\operatorname{Tr}\left(\phi \hat{f}_{\lambda}\right) \rightarrow^{\lambda} 0$.

Chapter 6. A proper containment of $W(\hat{G})$ in $\mathcal{L}^{\infty}(\hat{G})$. Let $Z$ be the integers and $Z_{+}$the positive integers. Denote by $\phi_{+}$the characteristic function of $Z_{+}$. Now $\phi_{+} \in l^{\infty}(Z)$ but is not w.a.p. One way to see this is to note that $\left\{R(-k) \phi_{+}\right\}_{k=1}^{\infty}$ converges pointwise to 0 but not quasi-uniformly. This idea will show for $G$ a complete direct product of a countably infinite collection of nontrivial compact groups that $W(\hat{G})$ is a proper closed subspace of $\mathcal{L}^{\infty}(\hat{G})$. 
Definition 6.1. Let $F \subset \hat{G}$. We say that $F$ is a $Q$-set if and only if there is a sequence $\left\{\alpha_{k}\right\} \subset \hat{G}$ such that:

(1) for $\alpha \in \hat{G},\left(\alpha_{k} \otimes \alpha\right) \cap F=\varnothing$ eventually,

(2) given $l \in Z_{+}$, there is $\alpha \in \hat{G}$ such that $\cup\left\{\alpha \otimes \alpha_{i}: 1 \leq i \leq l\right\} \subset F$.

The sequence $\left\{\alpha_{k}\right\}$ is called the associated sequence.

Remark 6.2. (a) Let $G=T$, the circle group. Then $F=Z_{+}$is a $Q$-set in $T=Z$ with associated sequence $\{-k\}_{k=1}^{\infty}$.

(b) Let $G=\prod_{n} Z\left(p_{n}\right)$, the complete direct product of a countable number of cyclic groups $Z\left(p_{n}\right)$ of order $p_{n}>1$. Then $F=\left\{x \in \hat{G}=\Sigma \bigoplus_{n} Z\left(p_{n}\right): x\right.$ $=\left(x_{1}, \ldots, x_{n}, 0,0, \ldots\right), x_{n} \neq 0, n$ even $\}$ is a $Q$-set in $G$ with associated sequence $\left\{x^{(k)}\right\}, x^{(k)}=(0, \ldots, 0,1,0, \ldots)$, where 1 appears in the $(2 k+1)$ th coordinate.

(c) Let $G=\prod_{n} G_{n}$, the complete direct product of a countable set of nontrivial compact groups. Then $F=\left\{x \in \hat{G}: x=\alpha_{1} \otimes \cdots \otimes \alpha_{n}, \alpha_{i} \in \hat{G}_{i}(1 \leq i \leq n)\right.$, $\alpha_{n} \neq\{1\}, n$ even $\}$ is a $Q$-set in $G$ with associated sequence $\left\{x^{(k)}\right\}, x^{(k)} \in$ $\hat{G}_{2 k+1} \backslash\{1\}$.

(d) Let $G=\Delta_{p}$ the $p$-adic integers. Then $\hat{G}=Z\left(p^{\infty}\right)$, the group of unimodular complex numbers of the form $x=\exp \left(2 \pi i l / p^{r+1}\right), 0 \leq l<p^{r+1}, l, r \in Z_{+}$. Then $F=\left\{x \in Z\left(p^{\infty}\right)\right.$ : ord $\left.x=p^{2 n}, n \in Z_{+}\right\}$is a $Q$-set in $Z\left(p^{\infty}\right)$ with associated sequence $x_{k}=\exp \left(2 \pi i / p^{2 k+1}\right)$.

Theorem 6.3. Let $F$ be a $Q$-set in $\hat{G}$ with assoicated sequence $\left\{\alpha_{k}\right\}$. Then $\phi \in \mathcal{L}^{\infty}(\hat{G})$ defined by $\phi_{\alpha}=I_{n_{\alpha}}$ (identity operator in $\mathcal{B}\left(C^{n_{\alpha}}\right)$ ) for $\alpha \in F$ and $\phi_{\alpha}=0$ otherwise, is not in $W(\hat{G})$.

Proof. Let $f_{k}=\chi_{a_{k}} / n_{a_{k}}$. Then $f_{k} \in P$. It follows from Propositions 4.2 and 4.7 that $\alpha \in \operatorname{spt}\left(f_{k} \cdot \phi\right)$ implies $\left(\alpha_{k} \otimes \alpha\right) \cap F \neq \varnothing$. Thus $f_{k} \cdot \phi \rightarrow^{k} 0$ pointwise on $\hat{G}$.

It remains to show that $\left\{f_{k} \cdot \phi\right\}$ has no subsequence which converges to 0 quasiuniformly. Now for $\psi \in \mathcal{Z} \mathcal{L}^{\infty}(\hat{G})$, we write $\psi_{a}=c_{\alpha}(\psi) I_{n_{k}}(\alpha \in \hat{G})$. Then for $\beta \in \hat{G}, \chi_{\beta} \cdot \psi \in \mathcal{L} \mathcal{L}^{\infty}(\hat{G})$ and a simple computation shows

$$
c_{\alpha}\left(\chi_{\beta} \cdot \psi\right)=\frac{1}{n_{\gamma}} \sum_{\gamma \in G} n_{\gamma} m_{\alpha \beta}(\gamma) c_{\gamma}(\psi)
$$

Now suppose there is $l \in Z_{+}$such that for each $\alpha \in \hat{G}, \min \left\{\left\|\left(f_{k} \cdot \phi\right)_{\alpha}\right\|_{\infty}: 1 \leq k\right.$ $\leq l\}<\frac{1}{2}$. By property 6.1(2), there is $\alpha_{0} \in \hat{G}$ such that $\left\{\alpha_{0} \otimes \alpha_{i}: 1 \leq i \leq l\right\}$ $C F$. But then we will have

$$
\begin{aligned}
\left\|\left(f_{k} \cdot \phi\right)_{a_{0}}\right\|_{b_{0}} & =\left|c_{a_{0}}\left(f_{k} \cdot \phi\right)\right| \\
& =\left|\frac{1}{n_{a_{0}} n_{\alpha_{k}}} \sum_{\gamma \in G} n_{\gamma} m_{a_{0} \alpha_{k}}(\gamma) c_{\gamma}(\phi)\right| \\
& =\frac{1}{n_{a_{0}} n_{\alpha_{k}}} \sum_{\gamma \in G} n_{\gamma} m_{a_{0} \alpha_{k}}(\gamma)=1,
\end{aligned}
$$


for $m_{\alpha_{0} \alpha_{k}}(\gamma) \neq 0$ only for $\gamma \in \alpha_{0} \otimes \alpha_{k} \subset F, c_{\gamma}(\phi)=1$ for $\gamma \in F$ and the identity $\sum_{\gamma \in G} n_{\gamma} m_{\alpha \beta}(\gamma)=n_{\alpha} n_{\beta}$, any $\alpha, \beta, \gamma \in \hat{G}$ (see Proposition 4.2).

Corollary 6.4. Let $G=\Pi_{n} G_{n}$ be the complete direct product of a countable collection of nontrivial groups. Then $W(\hat{G})$ is a proper closed subspace of $\mathcal{L}^{\infty}(\hat{G})$.

Corollary 6.5. Let $G$ be a nondiscrete locally compact abelian group. Then there is a continuous, bounded function on $\hat{G}$ which is not weakly almost periodic.

Proof. If $G$ is compact, then $\hat{G}$ is a discrete (infinite) abelian group and thus has a subgroup of the form: (a) $Z$, (b) $Z\left(p^{\infty}\right)$, (c) a countable infinite sum of cyclic groups (see [2, Chapter 2]). By Theorem 6.3 and Remark 6.2, our conclusion is valid provided $G$ is: (a) $T$, (b) $\Delta_{p}$, (c) a countably infinite product of cyclic groups. Observe that if the result is valid for $\Lambda$, a closed discrete subgroup of $\hat{G}$, then it is certainly valid for $\hat{G}$ since the restriction of a continuous w.a.p. function to $\Lambda$ is a w.a.p. function and every bounded function on $\Lambda$ extends to a continuous bounded function on $\hat{G}$. Thus the result holds for those $G$ for which $\hat{G}$ is discrete or has an infinite discrete closed subgroup. By the principal structure theorem $[10$, p. 40$]$ for 1.c.a. groups we may assume $\hat{G}$ has a nonempty compact open subgroup $\Lambda$ (otherwise $\hat{G}$ contains a euclidean space, thus $Z$ ). Since $\hat{G} / \Lambda$ is an infinite discrete group, we let $f$ be a bounded function on $\hat{G} / \Lambda$ which is not w.a.p. Extend $f$ to all of $\hat{G}$ by $f(y+\Lambda)=f(y), y \in \hat{G}$. Clearly $f$ is not w.a.p. on $\hat{G}$ (by quasi-uniform convergence).

Remark 6.6. Another proof of Corollary 6.4 may be found in $[1$, p. 68].

\section{EXTENSIONS OF THE THEORY}

Chapter 7. Almost periodic functionals. One may drop the word "weakly" from Definition 2.1 and thus create a nonabelian analogue of almost periodic functions. We use the notation of Chapter 2.

Definition 7.1. For $G$ a compact group and $\phi \in \mathcal{L}^{\infty}(\hat{G})$, we say that $\phi$ is almost periodic if and only if the map $f \mapsto f \cdot \phi$ from $A(G) \rightarrow \mathcal{L}^{\infty}(\hat{G})$ is a compact operator. The space of all such $\phi$ is denoted by $A P(\hat{G})$.

The following is clear (see Theorem 2.5 and $[4$, p. 486]).

Theorem 7.2. $A P(\hat{G})$ is a closed *-submodule of $W(\hat{G})$, and a priori of $\mathcal{L}^{\infty}(\hat{G})$.

Theorem 73. If $\mu$ is a discrete measure in $M(G)$ then $\hat{\mu} \in A P(\hat{G})$.

Proof. If $\mu$ is finitely supported then $f \mapsto f \cdot \hat{\mu}$ is of finite rank.

Chapter 8. Locally compact groups. All the numbered definitions, propositions, and theorems of Chapters 2 and 7 also hold for noncompact locally compact groups, if the following replacements are made:

(i) The Fourier algebra $A(G)$ is also defined for locally compact groups, and is a regular commutative Banach algebra of continuous functions on $G$ vanishing at infinity (see [6]). 
(ii) Replace $\mathcal{L}^{\infty}(\hat{G})$ by $V N(G)$, the weak-operator closed algebra of operators on $L^{2}(G)$ (left Haar measure) generated by the left translations. Each $\mu \in M(G)$ is represented in $V N(G)$ as a left convolution operator $f \mapsto \mu * f, f \in L^{2}(G)$. Again $V N(G)$ is the dual of $A(G)[6]$ and is an $A(G)$-module.

\section{BIBLIOGRAPHY}

1. R. Burckel, Weakly almost periodic functions on semigroups, Gordon and Breach, New York, 1970. MR 41 \#8562.

2. C. Dunkl and D. Ramirez, Topics in harmonic analysis, Appleton-Century-Crofts, New York, 1971.

3.—, Sidon sets on compact groups, Monatsh. Math. 75 (1971), 111-117.

4. N. Dunford and J. T. Schwartz, Linear operators. I: General theory, Pure and Appl. Math., vol. 7, Interscience, New York, 1958. MR 22 \#8302.

5. W. Eberlein, Abstract ergodic theorems and weak almost periodic functions, Trans. Amer. Math. Soc. 67 (1949), 217-240. MR 12, 112.

6. P. Eymard, L'algèbre de Fourier d'un groupe localement compact, Bull. Soc. Math. France 92 (1964), 181-236. MR 37 \#4208.

7. J. Kitchen, Normed modules and almost periodicity, Monatsh. Math. 70 (1966), 233-243. MR 33 \#6297.

8. R. Phelps, Lectures on Choquet's theorem, Van Nostrand, Princeton, N.J., 1966. MR 33 \# 1690.

9. D. Ramirez, Weakly almost periodic functions and Fourier-Stieltjes transforms, Proc. Amer. Math. Soc. 19 (1968), 1087-1088. MR 38 \# 488.

10. W. Rudin, Fourier analysis on groups, Interscience Tracts in Pure and Appl. Math., no. 12, Interscience, New York, 1962. MR 27 \#2808.

Department of Mathematics, University of Virginia, Charlottesville, Virginia 22903 